\title{
Use of Fluoride Dentifrices for Children: Evaluation of General and Pediatric Dentists' Recommendations
}

\section{Ana Paula Dias Moreno} \\ Universidade de Sao Paulo \\ Marina Moscardini Vilela \\ Universidade de Sao Paulo \\ José Tarcísio Lima Ferreira \\ Universidade de Sao Paulo \\ Maria da Conceição Pereira Saraiva \\ Universidade de Sao Paulo
}

Fernanda Souza Liévana ( $\sim$ fernanda.lievana@usp.br)

Universidade de Sao Paulo https://orcid.org/0000-0001-9995-5613

\section{Andiara De Rossi}

Universidade de Sao Paulo

Research article

Keywords: pediatric dentistry, preventive dentistry, fluoride dentifrice, oral hygiene.

Posted Date: October 13th, 2020

DOl: https://doi.org/10.21203/rs.3.rs-76149/v1

License: (1) This work is licensed under a Creative Commons Attribution 4.0 International License.

Read Full License 
USE OF FLUORIDE DENTIFRICES FOR CHILDREN: EVALUATION OF GENERAL AND PEDIATRIC DENTISTS' RECOMMENDATIONS 


\section{ABSTRACT}

Background: The aim of this study was to evaluate whether the recommendations of General Dentists (GD) and Pediatric Dentists (PD) on the use of fluoride dentifrice by children are in agreement with the recommendations given in dental association guidelines. Methods: A questionnaire with 11 questions was applied to GD and PD. Questions were about professional performance, as well as type and amount of dentifrices recommended according to the child's age. The way information was conveyed to patients and caregivers was also evaluated. The analysis of the associations between responses and professional training were performed using the Chi-square or Fisher's Exact tests. Results: 354 professionals filled out the questionnaire. The final response rates were $68 \%$ for GD and $85 \%$ for PD. GD had more recommendations that differ from those found in the guidelines than PD. Conclusion: There is still divergence between the guidelines recommended by dental associations with those of GD and PD. Therefore, it is necessary to conduct educational campaigns for all professionals in the oral health area.

Keywords: pediatric dentistry, preventive dentistry, fluoride dentifrice, oral hygiene. 


\section{INTRODUCTION}

Although the use of fluoride has resulted in a significant reduction in the prevalence and occurrence of caries disease in children [1-3] the ingestion of excessive doses of fluoride during dental development may lead to undesirable acute and chronic effects, such as dental fluorosis [4-6]. The risk of dental fluorosis occurring is higher due to the possibility of high absorption of fluoride, mainly due to the inadvertent ingestion of high doses of fluoridated toothpastes $[4,7]$.

In order to reduce the intake of fluoridated dentifrices by children and to prevent dental fluorosis, dentifrices without fluoride or with low fluoride (550ppmF) emerged. However, randomized controlled trials and systematic reviews have no found scientific evidence to recommend the use of fluoride-reduced dentifrices to prevent caries disease [3, 8, 9]. Currently, three main types of toothpaste for children are commercialized: fluoride-free; low-fluoride (400-500 ppmF) and conventional toothpaste (1100 ppmF). International and Brazilian associations present guidelines with prescriptions for each toothpaste according to children's age.

The current guidelines of American Academy of Pediatric Dentistry (AAPD) and American Association for Dental Research (AADR) recommend conventional fluoride dentifrices restricting the amount of fluoride according to age: smear size amount for children younger than two years old and pea-sized amount for children between ages two to five years old $[10,11]$. The guidelines also recommend adult supervision while children brush their teeth. The American Dental Association (ADA) recommends fluoride dentifrices with minimum content of $1100 \mathrm{ppmF}$ for children of all ages using a smear size amount or a rice-size amount for children under three years old, which corresponds to $0.1 \mathrm{mg}$ of fluoride. The amount is not to be greater than a pea-size for children between three to six years old, which corresponds to $0.25 \mathrm{mg}$ of fluoride [12]. In addition, the guideline also recommended adult supervision at brushing to ensure the use of appropriate amount of dentifrice. The ADA also emphasizes the importance of the professional verbal information given to parents and caregivers, followed by active demonstration of the amount of dentifrice to be used.

The European Academy of Paediatric Dentistry (EAPD) recommends that babies (up to 2 years old) and children (from 2 to 6 years old) use conventional fluoride dentifrice (1000 ppmF). The amount of 0.125 grams (grain of rice size) is recommended in babies and 0.25 grams (pea size) in children. The EAPD also suggests that children over 6 years old use toothpaste containing $1450 \mathrm{ppmF}$ covering the length of the toothbrush (0.5-1.0 grams) [13].

In Brazil, the Ministry of Health of Brazil (MS) recommends conventional fluoride dentifrice in small amounts (about 0.3 grams, equivalent to a grain of rice) for children less than nine years old [14]. MS does not recommend dentifrices with low fluoride or non-fluoride concentrations. The Guideline of Reference for Clinical Procedures in Pediatric Dentistry, Brazilian Association of Pediatric Dentistry (ABOPED) guideline, recommends conventional 
dentifrices with a minimum content of $1000 \mathrm{ppmF}$ for children of all ages. The amount of dentifrice recommended for children under three years old is the size of a rice grain (about $0.1 \mathrm{~g}$ ). The guide also recommends that children under six years old should be supervised by an adult while brushing and should avoid ingesting the dentifrice [15]. In spite of guidelines and protocols it is known that there is divergence between dentists recommendations [3].

Therefore, the objective of this study was to evaluate if the professional recommendations regarding the ideal fluoride dentifrice for children in different age groups are consistent with the recommendations given by the most National and International Oral Health Organizations.

\section{MATERIAL AND METHODS}

This study was approved by the Human Ethics Committee of the School of Dentistry of Ribeirão Preto. A questionnaire was composed of 11 multiple answer questions and was aiming to evaluate the recommendations of General and Pediatric Dentists on the use of toothpastes by babies and children. Questions were about professional performance, as well as type and amount of dentifrices recommended according to the child's age. The way information was conveyed to patients and caregivers as either verbal and/or demonstration applying toothpaste was evaluated. The questionnaire was previously tested in a pilot study with a sample of ten professionals with the goal to perform an analysis of the question's suitability.

Data was collected from a convenience sample composed of General Dentists (GD) ( $\mathrm{n}=400)$ graduates at School of Dentistry of Ribeirão Preto at University of São Paulo who graduated in the last five years and Pediatric Dentists (PD) $(n=110)$ post graduates who graduated in the past five years from the Department of Pediatric Dentistry at School of Dentistry of Ribeirão Preto at University of São Paulo. Mailing addresses were obtained and the questionnaire was sent to all GD and PD with a consent form by the graduate office and office of the Department of Pediatric Dentistry, respectively. Other data about the professionals (age, sex, graduation year and e-mail) are available in the university records. If there was no response, a new email was sent within 30 days. Thereafter it was considered unanswered. At the conclusion of the research the participating professionals received a copy of the work containing the results with the intention to raise awareness about the ideal recommendation of the use of dentifrice for babies and children.

A contingency table was made showing the absolute and relative frequencies of the answers to each question in relation to professional training (GD or PD). The analysis of the associations between responses and professional training were performed using the Chi-square or Fisher's Exact tests. All data analyzed was performed using the R program (R Foundation for Statistical Computing, Vienna, Austria), with a 5\% significance level. 


\section{RESULTS}

Four hundred questionnaires were distributed to general dentists and one hundred and ten were distributed to pediatric dentists. The final response rates were 68 percent $(\mathrm{N}=261)$ for the general dentists and 85 percent $(\mathrm{N}=93)$ for the pediatric dentists. In total, 354 providers responded to the questionnaire. Table 1 shows a comparison of the responses from both GD and PD.

There was a significant association between the responses regarding the dental care among children and the training of the professional ( $\mathrm{p}<0.05$ ). In the group of GD, $84 \%$ treat children eventually and $7 \%$ do not, whereas in the group of PD 67\% exclusively treat children. There was also a significant association between professional training and the response about toothpaste recommendations for children ( $\mathrm{p}<0.05)$. It is noted that $59 \%$ of GD and $87 \%$ of PD recommend toothpaste after the eruption of the first tooth. However, 34\% of GD and 13\% of PD surveyed still recommend the use of toothpaste once the child turns two. When asked about the type of toothpaste recommended for babies (from the first dental eruption up to 3 years old), it is noted that the majority of PD (74\%) recommended conventional fluoride, while the GD group opinions are divided between non-fluoride toothpaste (31\%), low fluoride (39\%) and conventional fluoride (30\%), $\mathrm{p}<0.05$. In both groups, $94 \%$ answered "smear" $(0.1 \mathrm{mg})$ when asked about the amount of toothpaste recommended for babies ( $p>0.05)$. However, both groups disagreed as to the amount recommended for children over the age of 4 years (p $<0.05)$. Most GDs $(81 \%)$ and PDs $(92 \%)$ answered "pea size" $(0.25 \mathrm{mg})$ as the recommended amount, but $19 \%$ of GD answered "smear" (0.1 mg) and 8\% of PD responded conventionally.

The GD were also asked about how the information on recommended toothpaste amount is provided to the child's legal guardian and the answers had a significant association with professional training (p <0.05). In both groups, most responded that they provide information both verbally and with a demonstration (60\% among GD and 65\% among PD), but the percentage of GD that only provide information verbally was lower (24\%) than among PD (12\%). In addition, PD use more demonstration techniques $(23 \%)$ than GD (16\%). There was also a significant association between the responses regarding the recommendation of adult supervision when children are brushing and the professional training of GD ( $<<0.05)$. The majority of GD (97\%) and PD (94\%) recommend supervision, but 3\% of GD responded "not applicable" and 6\% of PD “do not know”.

\section{DISCUSSION}

The use of fluoride toothpaste to reduce caries is strongly based on evidence [16] that is present in AAPD, ADA, AADR, EAPD, MS, and ABOPED guidelines. However, according to the data analyzed in this study, there is still a disagreement regarding toothpaste recommendations among dentists when compared to guidelines. A considerable number of GD (34\%) still recommend fluoridated toothpaste after the child is two years old. These responses go against 
International and National Organizations mentioned previously that recommend fluoridated toothpaste after the first tooth erupts. It is important to point out that to date there is no scientific basis to recommend that children should only use conventional fluoride toothpaste after a certain age because there is no association between the prevalence of dental fluorosis and use of fluoride toothpaste before such age, even for low fluoride content dentifrices [17]. Although fluoride dentifrices are considered a risk factor for fluorosis, the expectation in pediatric dentistry that the intake of dentifrices by young children increases the prevalence or severity of fluorosis does not justify its contraindication, as it is not scientifically substantiated. Therefore, educational action of Pediatric Dentists on this topic becomes necessary as it's an important aspect of our results. Our study found $74 \%$ of PDs surveyed recommend conventional fluoride toothpaste for babies and 94\% for children over four years old which aligns with National and International recommended guidelines.

It's already been proven there is no evidence for use of low-fluoride toothpaste $[8,18,19]$. However, the majority of GDs (39\%) recommend low-fluoride toothpaste for babies and 31\% recommend fluoride-free toothpaste. The majority of PDs (74\%) recommend conventional toothpaste for babies, followed by reduced (20\%) or without fluoride $(6 \%)$. These results show that divergences exist even among specialists on toothpaste recommendations according to organizational guidelines. All five association recommendations reviewed in this study recommend conventional fluoride toothpaste (1000 a 1500ppmF) for all children including babies. However, for babies that have a lower body mass and for children where there is a greater concern about the exposure dose, the use of minimal amounts of toothpaste is sufficient for the hygiene of erupted teeth.

All guidelines reviewed in this study recommend a restriction on the amount of toothpaste applied to toothbrushes based on age. This is an efficient way to minimize the risk of dental fluorosis in young children. When the amount of toothpaste is reduced, the dose of systemic exposure to fluoride is reduced without any prejudice of this preventive effect, considering the topical use of fluoride [20]. All professionals surveyed are in agreement with the recommended guidelines on the amount of toothpaste used for infants. A high number of PDs and GDs (94\%) recommend a "smear" (0.1 mg) for children up to three years old. Nevertheless, there is a need for educational action for both professionals and patients regarding the type of dentifrice and amount recommended for each child.

According to data from this study, most professionals demonstrate the amount of toothpaste to be put on a child's toothbrush in addition to providing verbal instructions to each patient, parents and/or caregiver. The International guidelines of the AAPD and ADA present figures to illustrate the amount recommended while the National guidelines of the Ministry of Health and ABOPED do not present images, just explanations. Although the AADR does not present an image, it mentions that the ADA recommendations should be followed. The ADA and ABOPED guidelines recommend that children's brushing should be performed under adult supervision. According to our results, the majority of GDs (97\%) 
and PDs (94\%) recommend that brushing by children should be supervised by an adult, indicating that all dentists are acting according to recommended guidelines.

An interesting piece of data in this study is that the majority (84.2\%) of professionals characterized as General Dentists eventually treat children while only $7.8 \%$ of GDs do not treat children. These results confirm the need for all dentists to know the correct recommendation of fluoridated dentifrices for children. In order to maintain the anti-caries benefit and reduce the risk of fluorosis by using conventional fluoride (1100 ppmF) toothpaste, it is safe to recommend its use by children of all ages if a small amount of dentifrice is used while brushing under adult supervision. It is essential that dentists seek knowledge and follow protocols aligned with National and International Oral Health Organizations recommended guidelines.

The authors accept that this study has some limitations as the low response rate limits the generalization of the results. However, the data provides a baseline for further investigation. Because the study was carried out with dentists trained at the same university, the applicability of the results can be limited. Therefore, it is possible that our findings are less generalizable if results were to be compared to other educational institutions assuming the same study was applied.

\section{CONCLUSION}

Based on the results of this study, there is still divergence between the guidelines recommended by dental associations with those of General and Pediatric Dentists surveyed. It is necessary to conduct educational campaigns for all professionals in the health area. Dentists' continual search for knowledge is essential to minimize divergences in recommendations when compared to current association guidelines.

\section{DECLARATIONS}

ABBREVIATIONS

GD: General Dentists

PD: Pediatric Dentists

\section{AVAILABILITY OF DATA AND MATERIALS}

The patients' data are not publically available in order to maintain them in strict confidence; however, it may be obtained from corresponding author on reasonable request.

\section{AUTHORS' CONTRIBUTIONS}

We declare that all authors included in this paper fulfill authorship criteria. FSL, AR and MCPS have worked in the conception and design of the study; FSL, AR, MCPS, APDM and JTLF have worked on the preparation of the 
questionnaire; FSL and MMV on data collection. FSL and AR have performed statistical data analyses and interpretation. FSL and AR have written the article. All authors have critically reviewed the manuscript and approved its last version for publication.

\section{ACKNOWLEDGEMENTS}

We thank the dentists who participated in the study. There was no funding. The authors have no financial relationships relevant to this article to disclose.

\section{ETHICS APPROVAL AND CONSENT TO PARTICIPATE}

This study was approved by the Human Ethics Committee of the Ribeirão Preto School of Dentistry (FORP USP), with the CAAE Protocol number: 56551716.3.0000.5419.

\section{CONSENT FOR PUBLICATION}

Not applicable.

\section{COMPETING INTERESTS}

The authors declare that they have no competing interests.

\section{REFERENCES}

1. Twetman S. Caries prevention with fluoride toothpaste in children: an update. Eur Arch Paediatr Dent. 2009;10:162-7.

2. Clark MB, Slayton RL. Fluoride use in caries prevention in the primary care setting. Pediatrics. 2014;134:626-33.

3. Walsh T, Worthington H V, Glenny A-M, Marinho VC, Jeroncic A. Fluoride toothpastes of different concentrations for preventing dental caries. Cochrane database Syst Rev. 2019;3:CD007868.

4. Levy SM. Review of fluoride exposures and ingestion. Community Dent Oral Epidemiol. 1994;22:173-80.

5. Bowen WH. Fluorosis: Is it really a problem? J Am Dent Assoc. 2002;133:1405-7.

6. Molina-Frechero N, Pierdant-Rodríguez AI, Oropeza-Oropeza A, Bologna-Molina R. Fluorosis and dental caries: an assessment of risk factors in Mexican children. Rev Investig Clin. 2012;64:67-73.

7. Lewis DW, Limeback H. Comparison of recommended and actual mean intakes of fluoride by Canadians. J Can Dent Assoc. 1996;62:708-9.

8. Ammari AB, Bloch-Zupan A, Ashley PF. Systematic review of studies comparing the anti-caries efficacy of children's toothpaste containing $600 \mathrm{ppm}$ of fluoride or less with high fluoride toothpastes of 1,000 ppm or above. Caries Res. 2003;37:85-92.

9. dos Santos APP, Nadanovsky P, de Oliveira BH. A systematic review and meta-analysis of the effects of fluoride toothpastes on the prevention of dental caries in the primary dentition of preschool children. Community Dent Oral Epidemiol. 2013;41:1-12. 
10. Dentistry AA of P. Guideline on fluoride therapy. Pediatr Dent. 2013;35:E165.

11. Ajiboye AS, Dawson III DR, Fox CH, Committee ASI. American Association for Dental Research policy statement on community water fluoridation. J Dent Res. 2018;97:1293-6.

12. Affairs ADAC on S. Fluoride toothpaste use for young children. J Am Dent Assoc. 2014;145:190.

13. Toumba KJ, Twetman S, Splieth C, Parnell C, van Loveren C, Lygidakis NA. Guidelines on the use of fluoride for caries prevention in children: an updated EAPD policy document. Eur Arch Paediatr Dent. 2019;20:507-16.

14. Brasil, Saúde M da, Saúde S de A à, Básica D de A. Guia de recomendações para o uso de fluoretos no Brasil. 2009.

15. Brazilian Association of Pediatric Dentistry. Use of Fluoride. In: Manual of Reference for Clinical Procedures in Pediatric Dentistry. 2017. p. 95-100.

16. Cury JA, Tenuta LMA. Evidence-based recommendation on toothpaste use. Braz Oral Res. 2014;28 SPE:1-7.

17. Davies RM, Ellwood RP, Davies GM. The rational use of fluoride toothpaste. Int J Dent Hyg. 2003;1:3-8.

18. Santos APP, Oliveira BH, Nadanovsky P. Effects of low and standard fluoride toothpastes on caries and fluorosis: systematic review and meta-analysis. Caries Res. 2013;47:382-90.

19. Zaze A, Dias AP, Amaral JG, Miyasaki ML, Sassaki KT, Delbem ACB. In situ evaluation of low-fluoride toothpastes associated to calcium glycerophosphate on enamel remineralization. J Dent. 2014;42:1621-5.

20. Ellwood RP, Cury JA. How much toothpaste should a child under the age of 6 years use? Eur Arch Paediatr Dent. 2009;10:168-74. 


\section{Supplementary Files}

This is a list of supplementary files associated with this preprint. Click to download.

- Table1.JPG 